\title{
Iridocorneal Endothelial Syndrome
}

National Cancer Institute

\section{Source}

National Cancer Institute. Iridocorneal Endothelial Syndrome. NCI Thesaurus. Code C84792.

A group of disorders characterized by the presence of an abnormal inner layer of the cornea. This abnormality results in iris distortion, corneal swelling, pupil distortion, and glaucoma. It usually affects only one eye. 